\title{
High-Harmonic Generation in Mott Insulators
}

\author{
Yuta Murakami, ${ }^{1}$ Martin Eckstein, ${ }^{2}$ and Philipp Werner ${ }^{1}$ \\ ${ }^{1}$ Department of Physics, University of Fribourg, 1700 Fribourg, Switzerland \\ ${ }^{2}$ Department of Physics, University of Erlangen-Nürnberg, 91058 Erlangen, Germany
}

(Received 25 December 2017; revised manuscript received 18 April 2018; published 2 August 2018)

\begin{abstract}
Using Floquet dynamical mean-field theory, we study the high-harmonic generation in the time-periodic steady states of wide-gap Mott insulators under ac driving. In the strong-field regime, the harmonic intensity exhibits multiple plateaus, whose cutoff energies $\epsilon_{\text {cut }}=U+m E_{0}$ scale with the Coulomb interaction $U$ and the maximum field strength $E_{0}$. In this regime, the created doublons and holons are localized because of the strong field and the $m$ th plateau originates from the recombination of $m$ th nearestneighbor doublon-holon pairs. In the weak-field regime, there is only a single plateau in the intensity, which originates from the recombination of itinerant doublons and holons. Here, $\epsilon_{\text {cut }}=\Delta_{\text {gap }}+\alpha E_{0}$, with $\Delta_{\text {gap }}$ the band gap and $\alpha>1$. We demonstrate that the Mott insulator shows a stronger high-harmonic intensity than a semiconductor model with the same dispersion as the Mott insulator, even if the semiconductor bands are broadened by impurity scattering to mimic the incoherent scattering in the Mott insulator.
\end{abstract}

DOI: 10.1103/PhysRevLett.121.057405

Introduction.-The interaction between intense laser fields and matter results in highly nonperturbative phenomena. Among them, the high-harmonic generation (HHG) is both interesting with regard to the underlying physical processes and in view of potential applications [1-4]. HHG in atomic and molecular gases has been intensively studied for decades and is the basis of attosecond science and new ultrafast imaging methods $[3,4]$. The recent observation of HHG in semiconductors has renewed the interest in this field [5-14]. Originating from the periodic arrangement of the atoms in solids, characteristic features of the HHG spectra, different from those of gases, have been observed. HHG in semiconductors can be used to explore the electron band properties $[5,7,11,12]$ and the lattice structure [13], to probe electron dynamics on ultrafast time scales $[9,11]$, and to develop new highfrequency laser light sources [11]. Theoretically, several mechanisms for HHG in solids have been proposed assuming weak correlations or an effective single-particle picture [5-25], such as intraband electron dynamics, interband contributions from electron-hole recombination $[9,18,19]$, and time-dependent diabatic processes $[14,20]$.

A different class of insulators in solid state physics is the Mott insulator (MI), which originates from strong electronic correlations, and the possibility of HHG in MIs has recently been pointed out [26,27]. In MIs, the excitation creates doublons and holons instead of electrons and holes in semiconductors, and their dynamics determines the current and the HHG. However, in contrast to semiconductors, excited charges cannot move freely in MIs because of Pauli blocking and scattering. Therefore, the features of the high-harmonic spectrum of MIs are not a priori clear, and the current understanding of HHG in MIs is very limited. Deeper insights into the underlying physics may lead to applications of HHG in the ultrafast imaging of the carrier dynamics in strongly correlated systems [26] and open a new class of materials for use in light sources.

In this Letter, we shed light on the periodically driven MI phase of the half-filled single-band Hubbard model. By means of the nonequilibrium dynamical mean-field theory (DMFT) [28], we reveal the general and fundamental structure of the HHG spectrum and its relation to the dynamics of the doublons and holons. Moreover, by comparing the HHG in MIs and semiconductor models, we find a different relation between the single particle spectrum and the HHG spectrum in these insulators.

Formalism. -We consider the Hubbard model attached to a thermal bath and driven by an ac field

$$
H=-\sum_{\langle i, j\rangle, \sigma} v_{i j}(t) c_{i, \sigma}^{\dagger} c_{j, \sigma}+U \sum_{i} n_{i \uparrow} n_{i \downarrow}+H_{\mathrm{bath}} .
$$

Here, $c_{i, \sigma}^{\dagger}$ is the creation operator of an electron at site $i$ with spin $\sigma, v_{i j}$ indicates the hopping parameter, $\langle i, j\rangle$ indicates the nearest-neighbor sites, $U$ is the interaction and $q$ is the charge. In the calculations, we use the gauge with pure vector potential $\mathbf{A}(t)$ so that the effect of the electric field $\mathbf{E}(t)$ appears in the phase of the hopping parameter $v_{i j}(t)=v_{i j} \exp \left[-i q \int_{\mathbf{r}_{i}}^{\mathbf{r}_{j}} d \mathbf{r} \mathbf{A}(t)\right]$, where $\mathbf{A}(t)$ is related to the electric field by $\mathbf{E}(t)=-\partial_{t} \mathbf{A}(t)$. This is equivalent to a pure scalar potential term $\sum_{i, \sigma} \Phi\left(\mathbf{r}_{i}, t\right) c_{i \sigma}^{\dagger} c_{i \sigma}=-\mathbf{E}(t)$. $\left(\sum_{i, \sigma} \mathbf{r}_{i} c_{i \sigma}^{\dagger} c_{i \sigma}\right)$ in the Hamiltonian. $H_{\text {bath }}$ represents a thermal bath of noninteracting electrons (the Büttiker model), which is introduced to describe the coupling of the system to an environment [29-31]. When the system is 

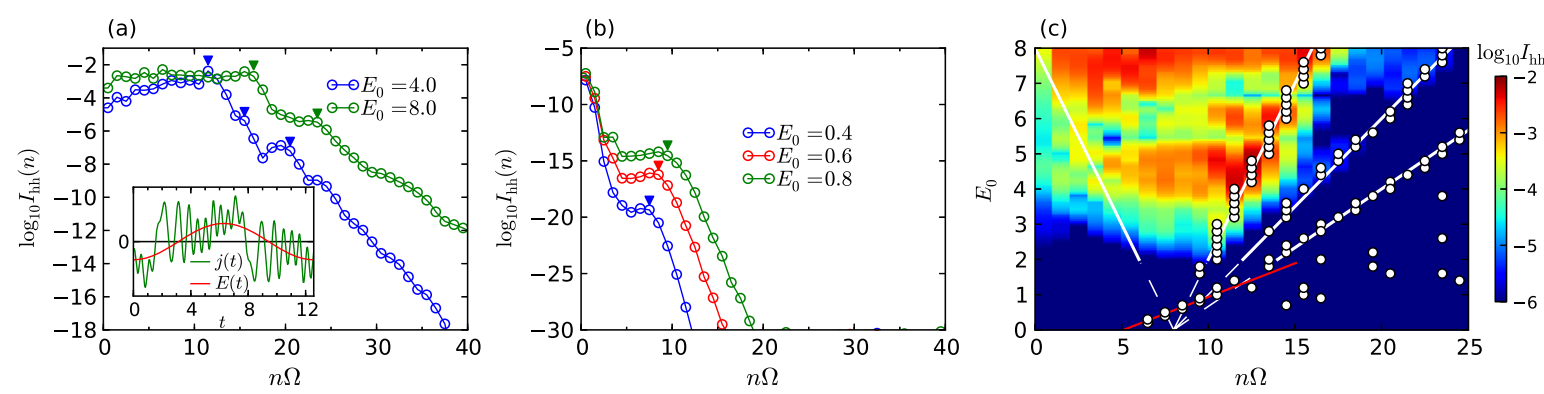

FIG. 1. (a) HHG spectra in the strong-field regime, (b) HHG spectra in the weak-field regime, and (c) HHG spectra as a function of the field strength $\left(E_{0}\right)$ and the harmonic energy $(n \Omega)$. The arrows and the white circle markers show cutoff energies. White lines in panel (c) indicate $n \Omega=U+m E_{0}$ and the red line is the fit for the weak-field regime. The inset of panel (a) shows the current and electric field during one period for $E_{0}=4.0$. We use $U=8.0, \beta=2.0, \Gamma=0.06, \Omega=0.5$.

continuously excited by an external field with frequency $\Omega$, it reaches a time-periodic nonequilibrium steady state (NESS) with a period $\mathcal{T} \equiv(2 \pi / \Omega)$, when the energy injected by the field is balanced by the dissipation to the bath.

We consider a hypercubic lattice with lattice spacing $a$ in the limit of infinite spatial dimensions $\left(v=\left[v^{*} /(2 \sqrt{d})\right]\right.$ with $d \rightarrow \infty$ ), which has a Gaussian density of states $\rho(\epsilon)=\left[1 /\left(\sqrt{\pi} v^{*}\right)\right] \exp \left[-\epsilon^{2} / v^{* 2}\right]$. The field is applied along the body diagonal, $\mathbf{A}(t)=A(t) \mathbf{e}_{0}$ with $\mathbf{e}_{0}=$ $(1,1, \ldots, 1)$ and $q a A(t)=A_{0} \sin \Omega t$, so that its strength along a given axis is $E(t)=-\left(A_{0} / q a\right) \Omega \cos \Omega \equiv$ $-E_{0} \cos \Omega t$. For the thermal bath, we employ the Büttiker model with a finite band width $W_{\text {bath }}$, $-\operatorname{Im} \Sigma_{\text {bath }}^{R}(\omega)=\Gamma \sqrt{1-\left(\omega / W_{\text {bath }}\right)^{2}}$. In the following, we set $q=1, a=1$ and use $v^{*}$ as the unit of energy. In order to clarify fundamental aspects of HHG in MIs, we focus on systems where the Mott gap is large compared to the width of the Hubbard bands, and the excitation frequency is much smaller than the gap. We typically use $U=8, \beta=2.0$, $\Gamma=0.06, W_{\text {bath }}=5, \Omega=0.5$.

To analyze the HHG spectrum, we focus on a NESS calculated within Floquet dynamical mean-field theory (FDMFT) [29-37]. We implement the FDMFT method with the noncrossing approximation (NCA) as an impurity solver $[38,39]$. NCA is the lowest order self-consistent hybridization expansion and is expected to produce qualitatively correct results for large $U$.

Results.-In Figs. 1(a) and 1(b), we show the HHG spectra in the strong field regime $\left(E_{0} \gtrsim 2\right)$ and the weak field regime $\left(E_{0} \lesssim 1\right)$. The HHG spectrum is evaluated from the square of the Fourier transformation of the dipole acceleration $(d / d t) j(t)$ as $I_{h h}(n \Omega)=|n \Omega j(n \Omega)|^{2}$ $[16,20,26]$ with $n \in \mathbb{Z}$, which is proportional to the power radiated at the given frequency. Here, the current is defined as $j(t)=i q \sum_{i, j, \sigma} v_{i j}(t)\left(\mathbf{e}_{0} \cdot \mathbf{r}_{i-j}\right)\left\langle c_{i, \sigma}^{\dagger}(t) c_{j, \sigma}(t)\right\rangle=\mathbf{e}_{0} \cdot \mathbf{j}(t)$ and $j(n \Omega)=(1 / \mathcal{T}) \int_{0}^{\mathcal{T}} d \bar{t} e^{i \bar{t} n \Omega} j(\bar{t})$. In the inset of Fig. 1, we show an example of the time evolution of the electric field and the induced current during one period. Because of the inversion symmetry, only odd frequency components appear in the HHG spectrum.
When the field is strong, the HHG spectrum initially increases with increasing order $n$ and exhibits a wide plateau, see Fig. 1(a). After this first plateau, the intensity suddenly drops, but other plateau structures exist at higher harmonic energies. On the other hand, when the field is weak, the HHG spectrum first drops and then shows a plateau, after which the intensity vanishes exponentially, see Fig. 1(b). In both regimes, the cutoff energies monotonically increase with increasing field strength.

In Fig. 1(c), we show the HHG spectra as a function of $E_{0}$ and the harmonic energy $(n \Omega)$. The cutoff energies of the plateaus are indicated by white markers [40]. The HHG spectra have nontrivial structures: (i) the intensity is strong in the triangular region $U-E_{0} \lesssim n \Omega \lesssim U+E_{0}$, (ii) there is an enhanced intensity around $E_{0}=U / 2=4$, and (iii) the intensity is suppressed for $5 \lesssim E_{0} \lesssim 6$. In the strong-field regime, the cutoff energy scales as $\epsilon_{\text {cut,m }}=U+m E_{0}$. On the other hand, in the weaker field regime, the cutoff energy of the first plateau $\epsilon_{\mathrm{cut}, 1}$ scales as $\epsilon_{\mathrm{cut}, 1}=\Delta+\alpha E_{0}$, where $\Delta(\neq U)$ is an offset and $\alpha>1$ is not an integer. These features are generic, as we confirmed by changing $\Omega$ and $U$ [39].

We now discuss the origin of the HHG in MIs. There are two contributions to the current: the doublon or holon hopping $\left(j_{\text {hop }}\right)$ and the doublon-holon recombination and/ or creation $\left(j_{\mathrm{rc}}\right)$ [39]. The former is analogous to the intraband current in a semiconductor, while the latter corresponds to the interband current, which represents recombination and/or creation of electron-hole pairs. One can approximately evaluate both contributions by means of a generalized tunneling formula for NESSs $[35,39,41]$, which works quantitatively very well in the parameter regime considered here. Such an analysis shows that the contribution from the recombination of doublons and holons $\left(j_{\mathrm{rc}}\right)$ dominates the current and is responsible for the plateaus both in the weak and strong field regimes [39].

In the strong field regime, this scenario of a dominant recombination-creation current is further supported by the fact that the cutoff energy is proportional to $U$ (the contribution from doublon or holon hopping should not depend on this energy scale [42]). Thus, we can argue that 

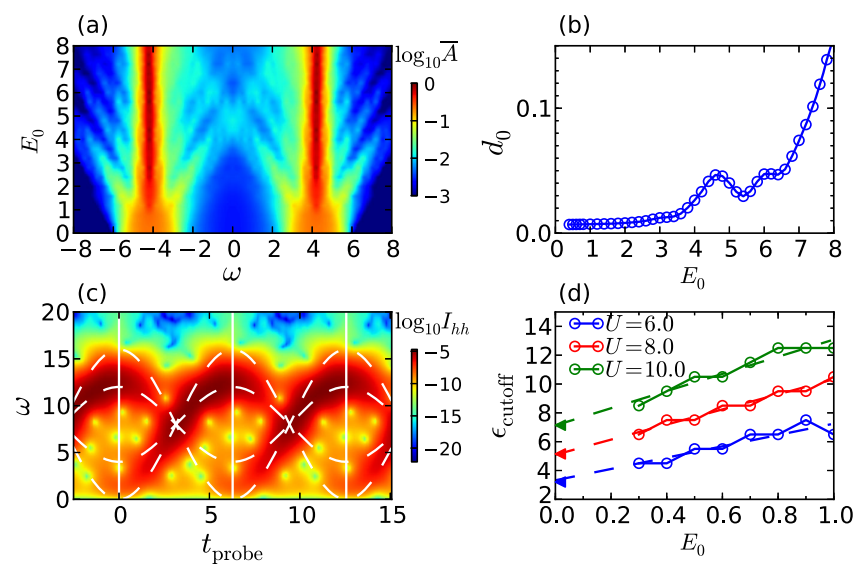

FIG. 2. (a) Time-averaged local spectral function $[\bar{A}(\omega)]$ of the nonequilibrium steady state as a function of $E_{0}$. (b) Field-strength dependence of the time-averaged doublon density $\left(d_{0}=\overline{\left\langle n_{\uparrow} n_{\downarrow}\right\rangle}\right)$ in the NESS. (c) Log-scale plot of the temporal HHG intensity $I_{h h}\left(\omega ; t_{\text {probe }}\right)$ for $\Omega=0.5, E_{0}=4.0$. The dashed lines are $\omega=U \pm m E(t)$. Vertical lines indicate $t_{\text {probe }}=0, \mathcal{T} / 2, \mathcal{T}$. Here, $U=8.0, \beta=2.0, \Gamma=0.06$, and $W_{\text {bath }}=5$. (d) Field-strength dependence of the cutoff energy in the weak-field regime for various $U$. Dashed lines are linear fits and the arrows at $E_{0}=0$ indicate the gaps estimated from the local spectral functions.

the different HHG plateaus originate from the recombination of a doublon-holon pair which is separated by $m$ sites: When $E_{0}$ is comparable or larger than the width of the Hubbard bands, doublons and holons remain almost localized. Indeed, the spectral functions in the NESS show clear Wannier-Stark peaks in the strong field regime [35,43], see Fig. 2(a). The recombination of a doublonholon pair separated by $m$ sites along the positive field direction releases the energy $U+m|E(t)|$ at a certain time $t$. This scenario consistently explains the main characteristic features of the HHG spectra in the strong-field regime. Since $E(t)$ oscillates between $-E_{0}$ and $E_{0}$, one expects that the light emitted from the recombination of an $m$ th nearestneighbor doublon-holon pair lies in the triangular region $U-m E_{0} \leq n \Omega \leq U+m E_{0}$, which naturally explains the prominent HHG in the dominant $m=1$ sector, and the weaker cutoffs at larger $m$. Second, when the doublon and holon density is small (large), there are less (more) doublons and holons to recombine, which leads to a low (high) HHG intensity. Indeed, we find that in the NESS the doublon (and holon) number is suppressed around $E_{0} \simeq 5.5$, see Fig. 2(b), which explains the valley in the HHG spectrum. The decrease of the double occupancy in the energy range $U / 2 \lesssim E_{0} \lesssim U$ comes from the absence of resonant tunneling processes. Similarly, the intense HHG spectrum around $E_{0}=U / 2=4$ is explained by an enhanced number of doublons. The peak in $I_{h h}$ and in the time-averaged doublon number is slightly shifted upward relative to $E_{0}=U / 2$. This can be explained by the oscillation of the field, which implies that larger field strengths are necessary for efficient tunneling to the next nearest neighbor site.

The scenario of recombination of $m$ th neighbor doublon-holon pairs is also confirmed by investigating the temporal structure of the HHG signal. We performed a windowed Fourier transformation of $j(t), j\left(\omega ; t_{\text {probe }}\right)=$ $\int d \bar{t} e^{i \bar{t} \omega} j(\bar{t}) W\left(\bar{t} ; t_{\text {probe }}\right)$ and evaluated $I_{\mathrm{hh}}\left(\omega ; t_{\text {probe }}\right) \equiv$ $\left|\omega j\left(\omega ; t_{\text {probe }}\right)\right|^{2}$. Here, $W\left(t ; t_{\text {probe }}\right)$ is the Blackman window function with a half-window of length 2 centered at $t=t_{\text {probe }}$. In Fig. 2(c), we show the result for $E_{0}=4.0$ on a logarithmic scale. The intensity peak at each $t_{\text {probe }}$ essentially follows $\omega=U \pm E(t)$, and we observe a sudden drop of the intensity near $\omega=U \pm 2 E(t)$. In particular, the $\omega=U+E_{0}$ and $\omega=U+2 E_{0}$ components are strong around $|E(t)|=E_{0}$, as expected from the scenario above.

In the weak-field regime $\left(E_{0} \lesssim 1\right)$, the cutoff scales as $\epsilon_{\text {cut }, 1}=\Delta+\alpha E_{0}$ with some noninteger constant $\alpha$, see Fig. 2(d). The offset $\Delta$, determined from extrapolations $E_{0} \rightarrow 0$, essentially coincides with the gap size [see arrows in Fig. 2(d)], which scales linearly with $U$. Again, this leads to the scenario that the HHG around the cutoff energy originates from doublon-holon recombination. In the weak-field regime, the almost unrenormalized spectrum [Fig. 2(a)] shows that the excited doublons and holons are not localized by the field and, thus, can move around the lattice to gain kinetic energy (ponderomotive energy $\left.E_{\text {kin }}\right)$ and emit this energy in the recombination process. This leads to emission at $n \Omega=\Delta_{\text {gap }}+E_{\text {kin }}$ in analogy with the three-step model for HHG in atoms and semiconductors $[1,2,19]$. Hence, the minimum emission energy from this process is $\Delta_{\text {gap }}$.

These results indicate that similar charge dynamics, as in semiconductors, also controls the HHG in MIs, despite the very different nature of these systems. In semiconductors, when the field is not too strong, the HHG is related to the recombination of itinerant electrons and holes in the valence and conduction bands [19], which yields a linear field dependence of the cutoff energy with an offset. In the strong-field regime, a quasistatic electric field analysis shows that the HHG originates from transitions among the localized Wannier-Stark states of the conduction and valence electrons, which results in multiple plateaus in the HHG spectrum [17].

In spite of these similarities, we now show that the relation between the single particle spectrum and the HHG intensity is very different in MIs and semiconductors. To this end, we study a semiconductor model with a valence band and a conduction band, corresponding to the upper and lower Hubbard band, respectively. Since, in the Hubbard model, the hopping of electrons in MIs leads to the creation of a doublon-holon pair at neighboring sites, we introduce the transfer integral between the different semiconductor orbitals at the neighboring sites. The resulting Hamiltonian reads 
(a)
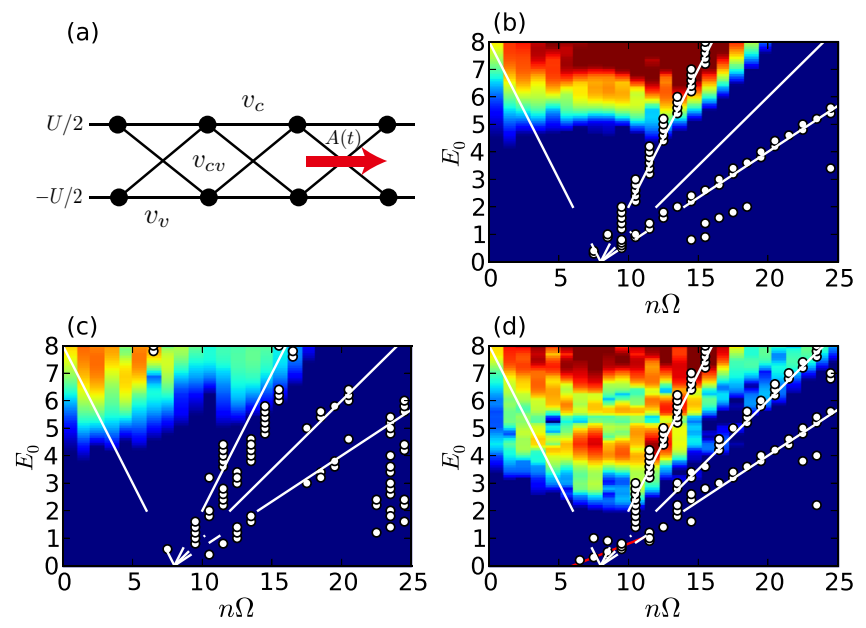

FIG. 3. (a) Schematic picture of the semiconductor model, Eq. (2). (b)-(d) HHG spectra $I_{h h}$ in the plane of $E_{0}$ and $n \Omega$. (b) Type 1 semiconductor model. (c) Same model with additional impurity scattering $V_{\mathrm{imp}}=0.55$. (d) Type 2 semiconductor model. The color scale is the same as in Fig. 2 and $\Omega=0.5$, $U=8, \beta=2.0, \Gamma=0.06$.

$$
\begin{aligned}
H_{\text {semi }}(t)= & -\sum_{\langle i, j\rangle, \alpha} v_{i j}^{\alpha}(t) c_{i \alpha}^{\dagger} c_{j \alpha}-\sum_{\langle i, j\rangle} v_{i j}^{c v}(t)\left(c_{i c}^{\dagger} c_{j v}+c_{i v}^{\dagger} c_{j c}\right) \\
& +\sum_{i, \alpha} D_{\alpha} c_{i \alpha}^{\dagger} c_{i \alpha},
\end{aligned}
$$

with $D_{\alpha}$ the band center for band $\alpha=\{v, c\}$, see Fig. 3(a). In order to mimic the Hubbard model, we choose $D_{v}=$ $-U / 2$ and $D_{c}=U / 2$. The effect of the electric field is included via the Peierls substitution, and we consider the NESS by attaching a Büttiker-type thermal bath. One reasonable way to determine the hopping parameters is to choose them such that the bands of the semiconductor model show a similar dispersion as the Mott insulator. In particular, when the Coulomb interaction is large compared to the hopping, one may naively expect that the dispersion is given by the Hubbard I (H1) approximation, which is based on the atomic-limit self-energy $\Sigma^{R}(\omega)=\left(U^{2} / 4 \omega\right)$ [44]. Then, the dispersion of the upper and lower Hubbard bands becomes $\epsilon_{\mathbf{k}, \pm}=\left(\epsilon_{\mathbf{k}} \pm \sqrt{\epsilon_{\mathbf{k}}^{2}+U^{2}}\right) / 2$, which is reproduced by choosing $v^{c}=v^{v}=v^{c v}=0.5 v$ (we call this the "type 1" model). The HHG spectrum of the type 1 semiconductor is shown in Fig. 3(b). The structure of the HHG spectrum is qualitatively very similar to that from the Hubbard I approximation [39], and one observes cutoff energies that scale with $U+E_{0}$ and $U+3 E_{0}$. However, the model underestimates the HHG spectrum in the weak to intermediate field regime, because electron-hole pairs are not efficiently created.

One major difference between the semiconductor model (or the H1 approximation) and the Hubbard model at finite $U$ is that it shows sharp peaks in $A(k, \omega)$, see Fig. 4. In the Hubbard model, even though the peak position of $A(k, \omega)$
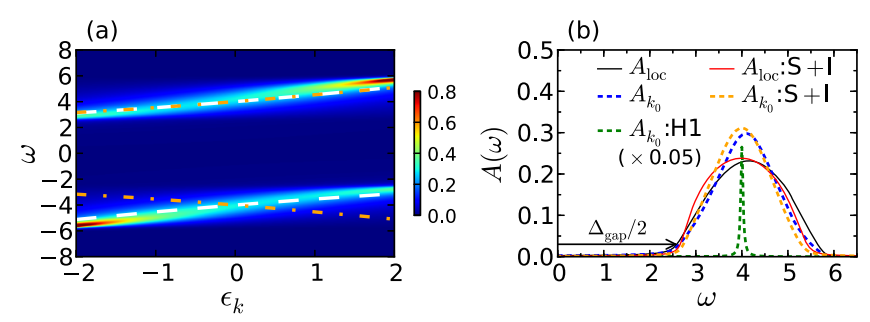

FIG. 4. (a) Momentum dependent spectral function $A(k, \omega)$ of the Mott insulator in equilibrium. The white lines show the peak position predicted by the $\mathrm{H} 1$ approximation and the type 1 semiconductor, while the dashed orange lines show the dispersion of the type 2 semiconductor. (b) Comparison between the local spectrum $A_{\mathrm{loc}}(\omega)$ and $A(k, \omega)$ at $\epsilon_{k}=0\left[A_{k_{0}}\right]$ obtained within DMFT, H1, and for the type 1 semiconductor with $V_{\text {imp }}=0.55$ $(\mathrm{S}+\mathrm{I})$.

at each $\epsilon_{k}$ roughly follows the prediction of the $\mathrm{H} 1$ approximation, there is a substantial width, comparable to the free electron band width, see Fig. 4(b). The incoherence originates from the charge dynamics in a random spin background and does not vanish in the limit $U \rightarrow \infty[39,45]$. The broadening of the single-particle spectrum can be reproduced in the type 1 semiconductor model by adding impurity effects through the self-energy $\Sigma_{\text {imp }}\left(t, t^{\prime}\right)=V_{\text {imp }}^{2} G_{\text {loc }}\left(t, t^{\prime}\right)$, as in Ref. [16], see Fig. 4(b). However, as shown in Fig. 3(c), the resulting HHG spectrum also does not reproduce the HHG spectrum of the MI. This implies that the strong high-harmonic signal of MIs is not simply related to the broadening of the bands.

Finally, we mention an interesting observation. The previously proposed three-step models and the strong-field theory have been discussed for semiconductors with a direct gap. By flipping the hopping of the valence band $v^{c}=-v^{v}=v^{c v}=0.5 v$ (we call this the "type 2" model), we realize a direct gap in our semiconductor model. In this case, the single particle dispersion is qualitatively different from the dispersion of the MI [Fig. 4(a)]. However, as shown in Fig. 3(d), the resulting HHG spectrum reproduces the qualitative features of the HHG spectrum of the MI. In the weaker field regime, the HHG spectrum has a unique plateau and the cutoff energy grows as $\alpha E_{0}$ with $\alpha>1$, while in the stronger field regime, there emerge multiple plateaus with cutoff $\epsilon_{\mathrm{cut}, m}=U+m E_{0}$. This model also reproduces the characteristic structures (i) to (iii) observed in the HHG spectrum of MIs.

Conclusions.-We revealed the general features of the HHG in wide-gap MIs under continuous ac driving. In the strong-field regime, the HHG spectra show multiple plateaus, which is explained by the recombination of localized doublons and holons at $m$ th nearest neighbor sites. In the weak-field regime, the HHG spectra show a single plateau, which comes from the recombination of itinerant doublonholon pairs. The different nature of MIs and semiconductors is reflected in a very different relation between the 
high-harmonic spectrum and the single particle spectrum, even though the HHG spectra become qualitatively similar under some conditions.

Strongly correlated systems are known for their high degree of tunability and exotic phases. In addition, they are a playground for photoinduced phase transitions between different phases. Therefore, they are an interesting platform to search for new sources of HHG, and ultrafast imaging based on HHG might be useful to understand the electron dynamics involved [26]. Developing a detailed understanding of the HHG profile in different classes of correlated materials is an interesting topic for future work.

The authors wish to thank D. Golež, Z. Lenarčič, M. Schüler, T. Oka, and N. Tsuji for fruitful discussions. This work was supported by the Swiss National Science Foundation through NCCR MARVEL and the European Research Council through ERC Consolidator Grant No. 724103. The calculations have been performed on the Beo04 cluster at the University of Fribourg, and the CSCS Dora cluster provided by MARVEL.

[1] P. B. Corkum, Phys. Rev. Lett. 71, 1994 (1993).

[2] M. Lewenstein, P. Balcou, M. Y. Ivanov, A. L'Huillier, and P. B. Corkum, Phys. Rev. A 49, 2117 (1994).

[3] A. L. Cavalieri et al., Nature (London) 449, 1029 (2007).

[4] F. Krausz and M. Ivanov, Rev. Mod. Phys. 81, 163 (2009).

[5] S. Ghimire, A. D. DiChiara, E. Sistrunk, P. Agostini, L. F. DiMauro, and D. A. Reis, Nat. Phys. 7, 138 (2011).

[6] O. Schubert et al., Nat. Photonics 8, 119 (2014).

[7] M. Hohenleutner, F. Langer, O. Schubert, M. Knorr, U. Huttner, S. W. Koch, M. Kira, and R. Huber, Nature (London) 523, 572 (2015).

[8] T. T. Luu, M. Garg, S. Yu. Kruchinin, A. Moulet, M. Th. Hassan, and E. Goulielmakis, Nature (London) 521, 498 (2015).

[9] G. Vampa, T. J. Hammond, N. Thiré, B. E. Schmidt, F. Légaré, C. R. McDonald, T. Brabec, and P. B. Corkum, Nature (London) 522, 462 (2015).

[10] F. Langer et al., Nature (London) 533, 225 (2016).

[11] G. Ndabashimiye, S. Ghimire, M. Wu, D. A. Browne, K. J. Schafer, M. B. Gaarde, and D. A. Reis, Nature (London) 534, 520 (2016).

[12] H. Liu, Y. Li, Y. S. You, S. Ghimire, T. F. Heinz, and D. A. Reis, Nat. Phys. 13, 262 (2016).

[13] Y. S. You, D. Reis, and S. Ghimire, Nat. Phys. 13, 345 (2017).

[14] N. Yoshikawa, T. Tamaya, and K. Tanaka, Science 356, 736 (2017).

[15] D. Golde, T. Meier, and S. W. Koch, Phys. Rev. B 77, 075330 (2008).

[16] A. F. Kemper, B. Moritz, J. K. Freericks, and T. P. Devereaux, New J. Phys. 15, 023003 (2013).

[17] T. Higuchi, M. I. Stockman, and P. Hommelhoff, Phys. Rev. Lett. 113, 213901 (2014).
[18] G. Vampa, C. R. McDonald, G. Orlando, D. D. Klug, P. B. Corkum, and T. Brabec, Phys. Rev. Lett. 113, 073901 (2014).

[19] G. Vampa, C. R. McDonald, G. Orlando, P. B. Corkum, and T. Brabec, Phys. Rev. B 91, 064302 (2015).

[20] T. Tamaya, A. Ishikawa, T. Ogawa, and K. Tanaka, Phys. Rev. Lett. 116, 016601 (2016).

[21] T. Otobe, Phys. Rev. B 94, 235152 (2016).

[22] T. T. Luu and H. J. Wörner, Phys. Rev. B 94, 115164 (2016).

[23] T. Ikemachi, Y. Shinohara, T. Sato, J. Yumoto, M. KuwataGonokami, and K. L. Ishikawa, Phys. Rev. A 95, 043416 (2017).

[24] N. Tancogne-Dejean, O. D. Mücke, F. X. Kärtner, and A. Rubio, Phys. Rev. Lett. 118, 087403 (2017).

[25] N. Tancogne-Dejean, O. D. Mücke, F. X. Kärtner, and A. Rubio, Nat. Commun. 8, 745 (2017).

[26] R. E. F. Silva, I. V. Blinov, A. N. Rubtsov, O. Smirnova, and M. Ivanov, Nat. Photonics 12, 266 (2018).

[27] N. Tancogne-Dejean, M. A. Sentef, and A. Rubio, arXiv:1712.01067.

[28] H. Aoki, N. Tsuji, M. Eckstein, M. Kollar, T. Oka, and P. Werner, Rev. Mod. Phys. 86, 779 (2014).

[29] N. Tsuji, T. Oka, and H. Aoki, Phys. Rev. B 78, 235124 (2008).

[30] T. Mikami, S. Kitamura, K. Yasuda, N. Tsuji, T. Oka, and H. Aoki, Phys. Rev. B 93, 144307 (2016).

[31] Y. Murakami, N. Tsuji, M. Eckstein, and P. Werner, Phys. Rev. B 96, 045125 (2017).

[32] P. Schmidt and H. Monien, arXiv:cond-mat/0202046.

[33] A. V. Joura, J. K. Freericks, and T. Pruschke, Phys. Rev. Lett. 101, 196401 (2008).

[34] N. Tsuji, T. Oka, and H. Aoki, Phys. Rev. Lett. 103, 047403 (2009)

[35] W.-R. Lee and K. Park, Phys. Rev. B 89, 205126 (2014).

[36] M. E. Sorantin, A. Dorda, K. Held, and E. Arrigoni, Phys. Rev. B 97, 115113 (2018).

[37] The time scale necessary to reach these steady states in a simulation starting from an equilibrium state depends on the strength of the bath coupling. For the parameters used in this Letter, this transient dynamics only takes a few cycles, so that it is meaningful to investigate the steady state directly.

[38] M. Eckstein and P. Werner, Phys. Rev. B 82, 115115 (2010).

[39] See Supplemental Material at http://link.aps.org/ supplemental/10.1103/PhysRevLett.121.057405 for the description of NCA, a tunneling formula and contributions to HHG from $j_{\text {hop }}$ and $j_{\text {rc }}$.

[40] The frequency $n \Omega$ is identified as a cutoff energy when $\ln I_{h h}(n \Omega)>\alpha \ln I_{h h}[(n-1) \Omega]$ and $\ln I_{h h}(m \Omega)<$ $\alpha \ln I_{h h}(n \Omega)$ for $m>n$. We use $\alpha=0.35$.

[41] Y. Murakami and P. Werner, Phys. Rev. B 98, 075102 (2018).

[42] Strictly speaking, the two processes cannot be fully decoupled and $j_{\text {hop }}$ can also be affected by the recombination or creation, which is also the case in the semiconductors $[18,19]$.

[43] P. Werner and M. Eckstein, Europhys. Lett. 109, 37002 (2015).

[44] J. Hubbard, Proc. R. Soc. A 276, 238 (1963).

[45] W. Metzner, P. Schmit, and D. Vollhardt, Phys. Rev. B 45, 2237 (1992). 